\title{
Soil biodegradation of a benzoxazinone analog proposed as a natural products-based herbicide
}

\author{
Nuria Chinchilla • David Marín • \\ Alberto Oliveros-Bastidas • José M. G. Molinillo • \\ Francisco A. Macías
}

Received: 17 October 2014 / Accepted: 20 April 2015 / Published online: 26 April 2015

(C) Springer International Publishing Switzerland 2015

\begin{abstract}
Aims Benzoxazinones with the 4-hydroxy- $(2 H)-1,4-$ benzoxazin-3(4H)-one skeleton have been proposed as potentially successful models for the development of novel design leads. D-DIBOA has proven to be the most promising base structure in the search for novel herbicide models based on the benzoxazinone skeleton. The biodegradation dynamics of D-DIBOA in soil are therefore relevant and are the subject of this study.

Methods A previously optimized methodology for the assessment of biodegradation has been applied for the first time to a synthetic benzoxazinone.

Results Biodegradability is a characteristic of natural benzoxazinones and a safety requirement for the development of herbicidal chemicals. The biodegradation phenomenon and its consequences for the development of new herbicide models are discussed. The half-life determined for D-DIBOA was much higher than those previously reported for the natural products DIBOA, DIMBOA and their benzoxazolinone derivatives.

Conclusions This finding, together with its previously described potent phytotoxicity, suggests that D-DIBOA is a useful candidate for novel herbicide model development. The lactam D-HBOA, which is slightly less
\end{abstract}

Responsible Editor: Leslie A. Weston.

N. Chinchilla $\cdot$ D. Marín · A. Oliveros-Bastidas ·

J. M. G. Molinillo · F. A. Macías $(\bowtie)$

Allelopathy Group, Department of Organic Chemistry, School of Sciences, Institute of Biomolecules (INBIO), University of Cadiz, C/ República Saharaui, n 7, Puerto Real, 11510 Cádiz, Spain

e-mail: famacias@uca.es phytotoxic than its precursor, was discovered to be the first and principal metabolite resulting from D-DIBOA degradation.

Keywords D-DIBOA · Benzoxazinones $\cdot$ Degradation study $\cdot$ Soil $\cdot$ Herbicide models

\section{Introduction}

Natural products have long been used as pesticides and have widely served as a source of inspiration for many commercial synthetic organic fungicides, herbicides and insecticides that are on the market today (Gerwick and Sparks 2014). One of the important benefits of natural products is that most of these compounds are rapidly degraded in the natural environment, a characteristic that accounts for the perception that most natural products are environmentally benign. However, this is possibly one of the Achilles' heels of natural products. The rate of degradation of natural products may also be too rapid to allow their development as successful herbicides (Dayan et al. 2012).

The possible application of benzoxazinones in the search for new natural herbicide models is a topic of current interest due to the wide variety of bioactivities and the ecological roles exhibited by natural benzoxazinones and some of their related compounds (Tables 1, 2) (Argandoña et al. 1983; Barnes and Putnam 1987; Bravo and Lazo 1996; Perez and Ormeno-Nunez 1991; Schulz et al. 2013). 
Table 1 Natural benzoxazinones and synthetic derivatives mentioned in this study

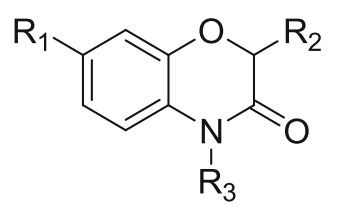

\begin{tabular}{lllll}
\hline & $\mathrm{R}_{1}$ & $\mathrm{R}_{2}$ & $\mathrm{R}_{3}$ & Acronym \\
\hline Natural & $\mathrm{H}$ & $\mathrm{O}-\beta-\mathrm{D}-\mathrm{Glc}$ & $\mathrm{OH}$ & DIBOA-Glc \\
Allelochemicals & $\mathrm{H}$ & $\mathrm{OH}$ & $\mathrm{OH}$ & DIBOA \\
(A) & $\mathrm{OCH}_{3}$ & $\mathrm{OH}$ & $\mathrm{OH}$ & DIMBOA \\
Degradation & $\mathrm{H}$ & $\mathrm{OH}$ & $\mathrm{H}$ & HBOA \\
Products & $\mathrm{OCH}_{3}$ & $\mathrm{OH}$ & $\mathrm{H}$ & HMBOA \\
(B) & & $\mathrm{H}$ & $\mathrm{H}$ & D-HBOA \\
Synthetic analogs & $\mathrm{H}$ & $\mathrm{H}$ & D-HMBOA \\
(C) & $\mathrm{OCH}_{3}$ & $\mathrm{H}$ & $\mathrm{OH}$ & D-DIBOA \\
& $\mathrm{H}$ & $\mathrm{H}$ & $\mathrm{OH}$ & D-DIMBOA \\
& $\mathrm{OCH}_{3}$ & $\mathrm{H}$ & $\mathrm{OAc}$ & ABOA \\
& $\mathrm{H}$ & $\mathrm{H}$ & $\mathrm{OAc}$ & AMBOA \\
\hline & $\mathrm{OCH}_{3}$ & $\mathrm{H}$ & & \\
\hline
\end{tabular}

Given the potential for the development of natural herbicide models of some of the synthetic benzoxazinone derivatives (Macias et al. 2005a, b, 2006b), it is necessary to characterize the behavior of such derivatives in a natural ecosystem. Laboratory studies on the phytotoxic effects of some synthetic derivatives of the benzoxazinones DIBOA and DIMBOA yielded promising results and these compounds therefore represent new leads for the development of chemicals that would be useful in weed control as natural templates.

From the point of view of the ecological role and potential applicability of benzoxazinones and related compounds in the development of novel herbicide models, it is interesting to characterize the biodegradability of these chemicals in soil (Cipollini et al. 2012). The stability of benzoxazinones and their derivatives has been extensively investigated, especially for natural benzoxazinones and benzoxazolinones (Fomsgaard et al. 2004; Macías et al. 2007; Oliveros-Bastidas et al. 2009).

As the degradation processes yield a wide variety of chemical structures, each one with different phytotoxic effects, the degradation phenomena observed for plant phytotoxins have interesting ecological implications (Macías et al. 2007). In fact, hydroxamic acids with the benzoxazinone skeleton yield lactams (Table 1), benzoxazolinones, aminophenoxazines, malonamic acids, aminophenols and acetamidophenols (Table 2) in different environments and under different conditions (Oliveros-Bastidas 2006). The presence and relative proportions of these chemicals in soil affects the chemoecological interactions between donor and target species, as demonstrated by recent findings (Macias et al. 2014).

The phytotoxicity of benzoxazinones and their degradation products on Standard Target Species (Macias et al. 2005b) and common weeds (Macias et al. 2005a, 2006b) has been evaluated. These reports offer a systematic comparison, under the same conditions, of benzoxazinones (hydroxamic acids and lactams), benzoxazolinones, aminophenoxazines, malonamic acids and aminophenols with a commercial herbicide (terbutryn $59.4 \%$, triasulfuron $0.6 \%$ ). The relative phytotoxicity was presented for each species and the synthetic benzoxazinones 4-hydroxy-(2H)-1,4-benzoxazin-3(4H)-one (D-DIBOA) and 4-acetoxy-(2H)-1,4benzoxazin-3(4H)-one (ABOA) were found to be the most phytotoxic (Fig. 1).

The lack of a hydroxyl group at position C-2 for these synthetic benzoxazinones, which was found to be closely associated with an increase in phytotoxicity, was proposed to enhance the stability of the benzoxazinone skeleton. The degradation process from benzoxazinones to benzoxazolinones is related to hemiketal cleavage at $\mathrm{C}-2$ in aqueous solution, in which the rate of this process is closely related to $\mathrm{pH}$ (Macias et al. 2005a, b, 2006b).

The presence of a hydroxyl group at $\mathrm{C}-2$ is the key for all DIBOA and DIMBOA degradation mechanisms. Thus, 2-deoxybenzoxazinones such as D-DIBOA could show very different degradation behavior and different metabolites could therefore appear in the soil after their application as pest management tools, which in turn could have potential consequences on their efficacy and environmental side-effects. In the work described here, degradation experiments were carried out in wheat crop soil and D-DIBOA was evaluated using previously reported methodologies for analysis (Eljarrat et al. 2004) and degradation kinetics (Macias et al. 2004, 2005c).

The choice of D-DIBOA as a model for phytotoxic benzoxazinones resulted in significant improvements in phytotoxicity and selectivity. The systematic esterification of D-DIBOA at the N-4 or C-2 positions resulted in a new generation of esters with increased lipophilicity. Stronger phytotoxic effects and higher selectivity were observed for these compounds in comparison to natural benzoxazinones (Macías et al. 2008; Macias et al. 
Table 2 Benzoxazinone degradation products discussed in this study

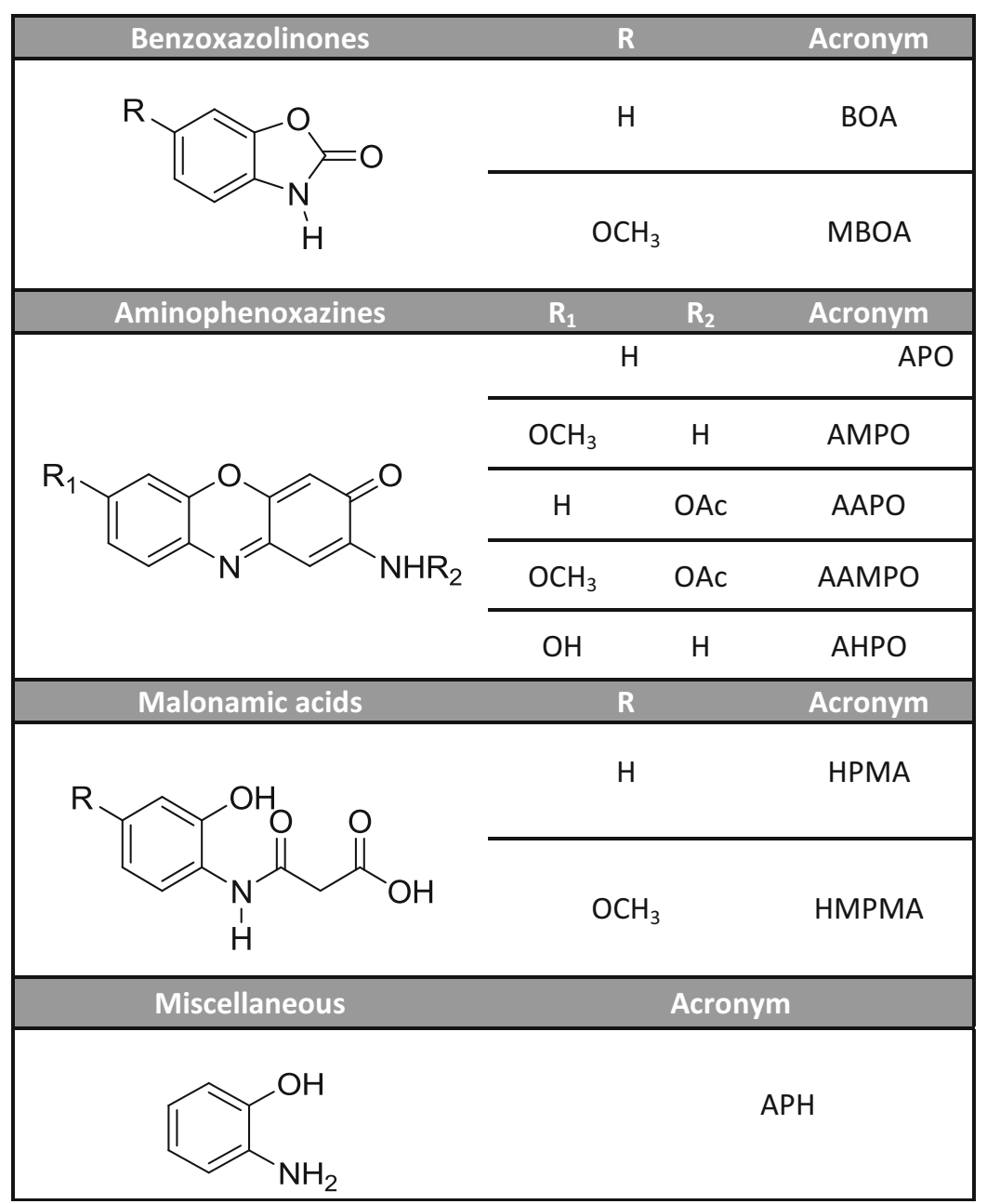

2006d). Among them, 4-propanoyloxy-(2H)-1,4-benzoxazin-3(4H)-one (Pr-D-DIBOA) and 2-ethyl-( $2 H)$ 1,4-benzoxazin-3(4H)-one (2-Et-D-DIBOA) were proposed as the optimal models for phytotoxic action and selectivity. These aspects were assessed by statistically comparing their effects on Avena fatua and Echinocloa crus-galli with those recorded on wheat and rice (Fig. 2).<smiles>O=C1COc2ccccc2N1O</smiles>

D-DIBOA<smiles>CC(=O)ON1C(=O)COc2ccccc21</smiles>

ABOA
Fig. 1 Structures of 4-hydroxy-(2H)-1,4-benzoxazin-3(4H)-one (D-DIBOA) and 4-acetoxy-(2H)-1,4-benzoxazin-3(4H)-one (ABOA)
Furthermore, improvement of the benzoxazinone skeleton by modification of the steric and electronic features was carried out (Macias et al. 2006a, 2009). Halogenated models 6-chloro-(2H)-1,4-benzoxazin3(4H)-one (6Cl-D-DIBOA), 6-fluoro-(2H)-1,4-benzoxazin-3(4H)-one (6 F-D-DIBOA) and 7-fluoro- $(2 H)-1,4-$ benzoxazin-3(4H)-one (7 F-D-DIBOA) were chosen for their phytotoxic effects, with the latter compound having a remarkable $A$. fatua-wheat selectivity, by comparison of their IC50 values on each species bioassay (Macias et al. 2006a). Optimal ranges for molecular volume, dipole moment and polarizability of benzoxazinones to maximize the phytotoxic activity were characterized (Fig. 2). Finally, considering the promising results obtained for some of the derivatives, the synthesis and bioactivity evaluation of models including more than one kind of structural modification 


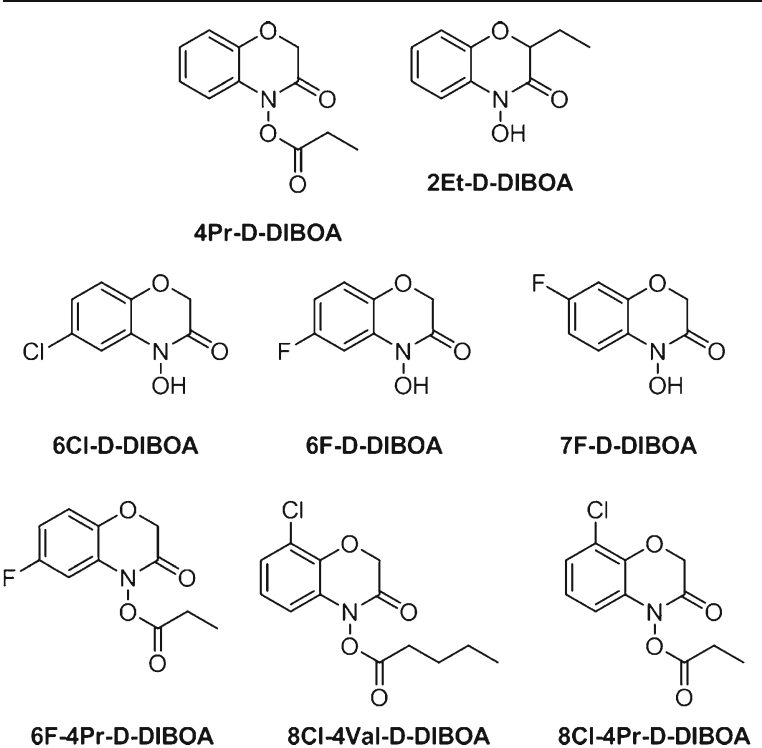

Fig. 2 Structures of the more active synthetic derivatives of D-DIBOA

were planned. In this respect, modification of the aromatic ring was combined with the modulation of lipophilicity in positions N-4 or C-2. 6-Fluoro-4propionyloxy-(2H)-1,4-benzoxazin-3(4H)-one (6 F4Pr-D-DIBOA), 8-chloro-4-valeryloxy-( $2 H)$-1,4-benzoxazin-3 $(4 H)$-one (8Cl-4Val-D-DIBOA) and 8chloro-4-propionyloxy-( $2 H)$-1,4-benzoxazin-3(4H)one (8Cl-4Pr-D-DIBOA) were the derivatives that showed the best bioactivity and selectivity profiles in the systems A. fatua-wheat and E. crus-galli-rice (Fig. 2) (Macias et al. 2010; Macías et al. 2010).

These findings suggest that D-DIBOA and its derivatives have great potential as agrochemicals. It is therefore of considerable interest to assess the stability of these compounds in soil cultures in the context of their possible use as models for the development of new herbicides. The biodegradation phenomena for D-DIBOA and their consequences are discussed in this work.

\section{Material and methods}

Soil sampling and experiment design

Soil samples were collected randomly from a cultivated crop field located at La Barca de la Florida, Cádiz, Spain $\left(36^{\circ} 38^{\prime} 28.18^{\prime \prime} \mathrm{N}, 5^{\circ} 57^{\prime} 43.14^{\prime \prime} \mathrm{W}\right)$. The samples were taken from the vicinity of the radicular system, with a maximum horizontal distance of $15 \mathrm{~cm}$ from the shoot, and a maximum depth from the ground surface of $10 \mathrm{~cm}$. There were no other plant species present in the soil at this time. The wheat was in its $\mathrm{N}^{\circ} 59$ ('end of heading') growth stage according to the $\mathrm{BBCH}$ growth stage classification, approximately 250 days after planting (Meier 1997). The collected soil samples were stored in plastic bags at $-15{ }^{\circ} \mathrm{C}$ prior to use. Before degradation experiments were performed, wheat soil was maintained at ambient temperature and dried for 2 days. All remaining vegetation and stones were removed manually and soil was sieved $(<1 \mathrm{~mm})$ and placed into $50 \mathrm{~mL}$ sterile glass vials (2 $\mathrm{g}$ each). One $\mathrm{mL}$ of a $2 \mathrm{mg} / \mathrm{mL}$ D-DIBOA sterile solution in deionized water (filter sterilization, $<2 \mu \mathrm{m}$ ) was placed into each vial. Two control sets were used: one set without soil to assess non-biocatalyzed degradation ('witness solution') and one with sterile soil (previously autoclaved) to record the influence of inorganic soil composition on degradation rates. All samples were placed in a growth chamber $\left(25^{\circ} \mathrm{C}, 16 \mathrm{~h}\right.$ light $/ 8 \mathrm{~h}$ darkness) for the duration of the experiment. Sterile and non-sterile samples, together with an aliquot of 'witness solution', were collected at $0,2,4,6,8,10$, 12 and 29 days (three replicates for each sampling time). After collection, $1 \mathrm{~mL}$ of $\mathrm{MeOH}$ was added to each sample to avoid subsequent microbial degradation. The samples were stored at $-15^{\circ} \mathrm{C}$ prior to analysis.

Analytical methodology and standardization

For HPLC analysis, soil solutions were centrifuged in a Selecta Microfiger BL 71379 apparatus at 13,000 rpm for $10 \mathrm{~min}$ and then filtered $(<44 \mu \mathrm{m})$. The resulting solid residue was extracted with methanol $(10 \mathrm{~mL})$ in an ultrasonic bath $\left(15 \mathrm{~min}, 5^{\circ} \mathrm{C}\right)$. The extract was centrifuged again for $10 \mathrm{~min}$ and the process was repeated three more times. This process was repeated using ethyl acetate $(10 \mathrm{~mL})$ as solvent. The soil aqueous solution, the methanol, and the ethyl acetate extracts were distilled under reduced pressure. The solid residues were dissolved in $\mathrm{MeOH}(2 \mathrm{~mL})$ with $1 \%$ acetic acid and then filtered $(<0.2 \mu \mathrm{m})$ prior to HPLC injection. All samples were analyzed on a Merck HITACHI HPLC system equipped with a LaChrom L-7100 quaternary gradient pump, an L-7455 LaChrom diode array detector and an L-7200 LaChrom autoinjector. Data were collected and processed using an HPLC data system (Merck HITACHI D7000). Instrument conditions for analysis were: Lichrospher $100 \mathrm{RP}-18(250 \times 4.0 \mathrm{~mm}$, 
Table 3 Calibration curves for the DIBOA degradation series

\begin{tabular}{lllllc}
\hline \multicolumn{2}{l}{ Abs $=A$ [chemical] $+B$} & & & \\
\hline Chemical & $\lambda_{\max }$ & $\mathrm{A}$ & $\mathrm{B}$ & $\mathrm{R}^{2}$ & $\mathrm{RT}(\mathrm{min})$ \\
\hline D-DIBOA & 280 & $2 \cdot 10^{7}$ & $10^{6}$ & 0.9854 & 14.75 \\
D-HBOA & 280 & $2 \cdot 10^{7}$ & $10^{6}$ & 0.9862 & 15.55 \\
DIBOA & 253 & $2 \cdot 10^{7}$ & 175074 & 0.9990 & 9.95 \\
BOA & 264 & $10^{7}$ & $2 \cdot 10^{6}$ & 0.9681 & 14.67 \\
APH & 269 & $10^{7}$ & 60279 & 0.9995 & 3.44 \\
HPAA & 278 & $10^{7}$ & 642363 & 0.9912 & 10.48 \\
APO & 260 & $3 \cdot 10^{7}$ & 241476 & 0.9990 & 22.53 \\
\hline
\end{tabular}

$5 \mu \mathrm{m})$ reversed-phase column at $25^{\circ} \mathrm{C}$. Mobile phases were water:1 $\% \mathrm{AcOH}(\mathrm{A})$ and methanol:1 $\% \mathrm{AcOH}$

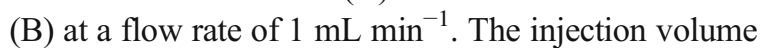
was $50 \mu \mathrm{L}$. The following gradient was used for separation: at $0 \mathrm{~min}, 30 \% \mathrm{~B} ; 2 \mathrm{~min}, 30 \% \mathrm{~B} ; 19 \mathrm{~min}, 60 \%$ B; $21 \mathrm{~min}, 100 \% \mathrm{~B}$. Under these conditions, the DDIBOA retention time was $14.75 \mathrm{~min}$. A calibration curve was created for D-DIBOA at $\lambda=280 \mathrm{~nm}(1,0.5$, 0.25 , and $0.125 \mathrm{mg} / \mathrm{mL})$. The equation for the curve was $\mathrm{Abs}=2 \cdot 10^{7}[\mathrm{D}-\mathrm{DIBOA}]+1 \cdot 10^{6}\left(R^{2}=0.9854\right)$.

In order to identify degradation products from $D$ DIBOA, several metabolites belonging to the natural DIBOA degradation series, together with lactam D-
Fig. 3 Top: D-DIBOA

chromatogram with UV detection at $280 \mathrm{~nm}$, and UV-vis spectrum. Bottom: Variation of D-DIBOA concentration with time
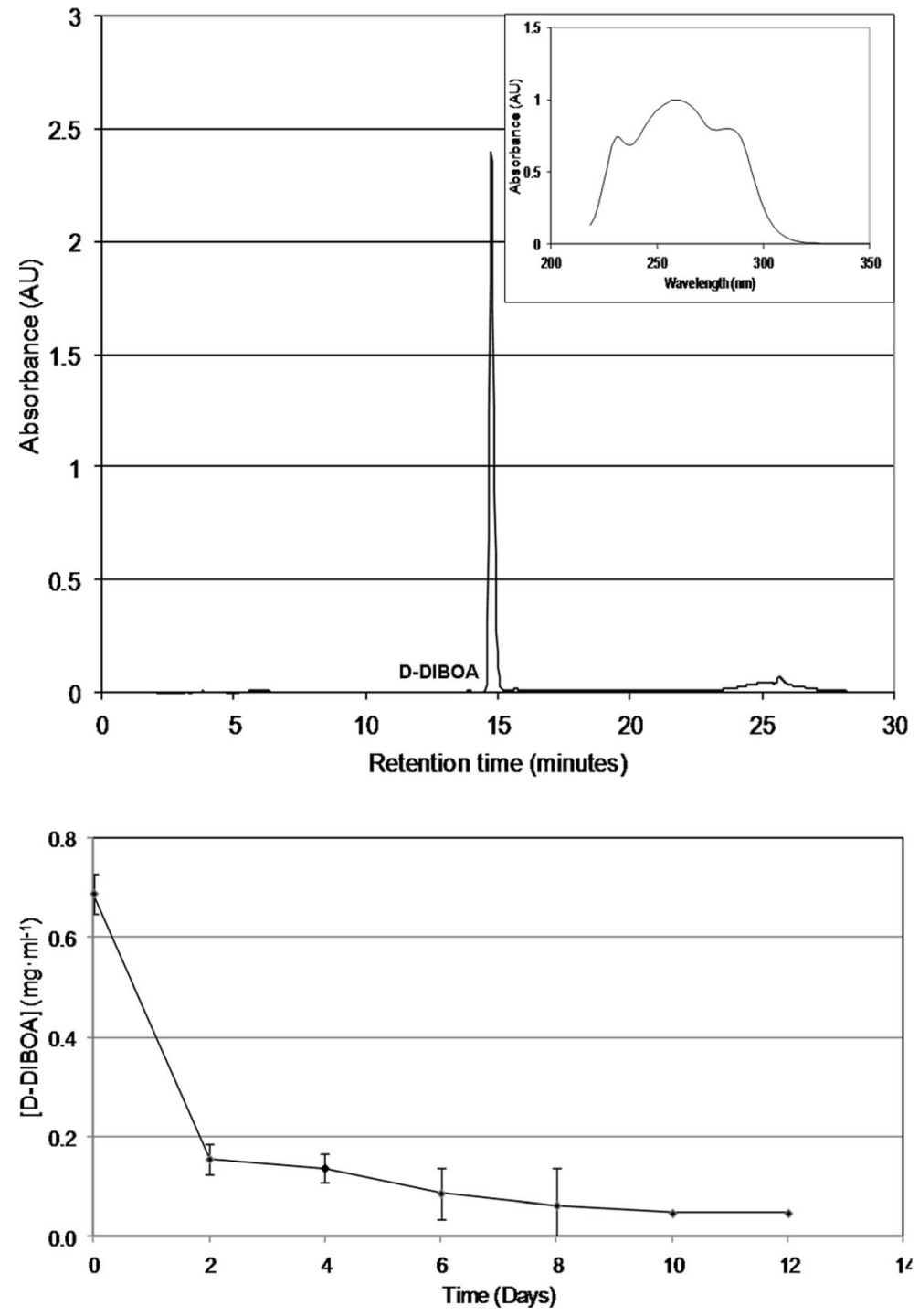
Fig. 4 Average regression curve for D-DIBOA concentrations

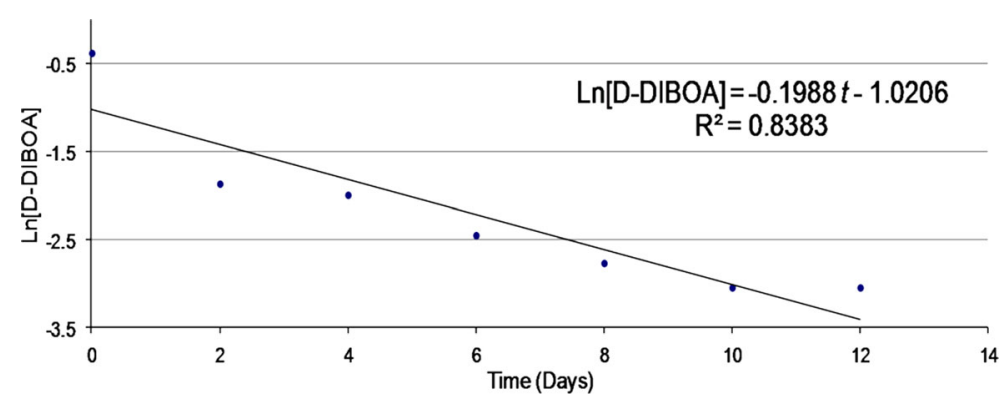

HBOA, were injected under the same conditions and the corresponding calibration curves were built (Table 3 ).

2-Aminophenol (APH) and 2-acetamidophenol (HPAA) were purchased from Sigma-Aldrich Co. and were used in assays. BOA was obtained from Fluka Chemika and was used as received. D-DIBOA, DHBOA and APO were obtained by the synthetic methods previously described by (Macias et al. 2006c).

D-DIBOA was degraded in wheat crop soil and its half-life in this environment was determined on the basis of the data points from the three determinations, treated independently. The structure of the main degradation product (D-HBOA) is proposed on the basis of its spectrophotometrical properties and by comparison with the synthetic standard.

\section{Results}

Degradation of D-DIBOA with time

The evolution of D-DIBOA concentration with time is shown in Fig. 3 together with a standard chromatogram and the UV-vis spectrum. Degradation was not observed in sterile control samples or in the witness solution.

The concentration data show a very steep decay in the D-DIBOA concentration from $t=0$ to $t=2$ days. The concentration continued to decay up to the 12th day. After this period, the system reached equilibrium and the concentration remained constant. The linear decay range to fit the data to $1 \mathrm{st}$ order kinetics was established from $\mathrm{t}=2$ to $\mathrm{t}=12$ days. The calculations provided $\mathrm{a}$ half-life $t_{1 / 2}=3.6 \pm 0.2$ days. The average regression curve is shown in Fig. 4. The average recovery of DDIBOA at $\mathrm{t}=0$ was $0.69 \pm 0.04 \mathrm{mg} \cdot \mathrm{mL}^{-1}$.

\section{D-DIBOA degradation metabolites}

Only one of the compounds chosen as potential degradation chemicals (see Table 1) was detected in the DDIBOA degradation experiment, namely lactam DHBOA, which was detected after 48 h (Fig. 5).

The evolution of D-HBOA concentration with time is shown in Fig. 6. A slight increase in concentration was observed up to day 19. After this time, the concentration
Fig. 5 D-DIBOA and D-HBOA signals, D-HBOA UV-vis spectrum

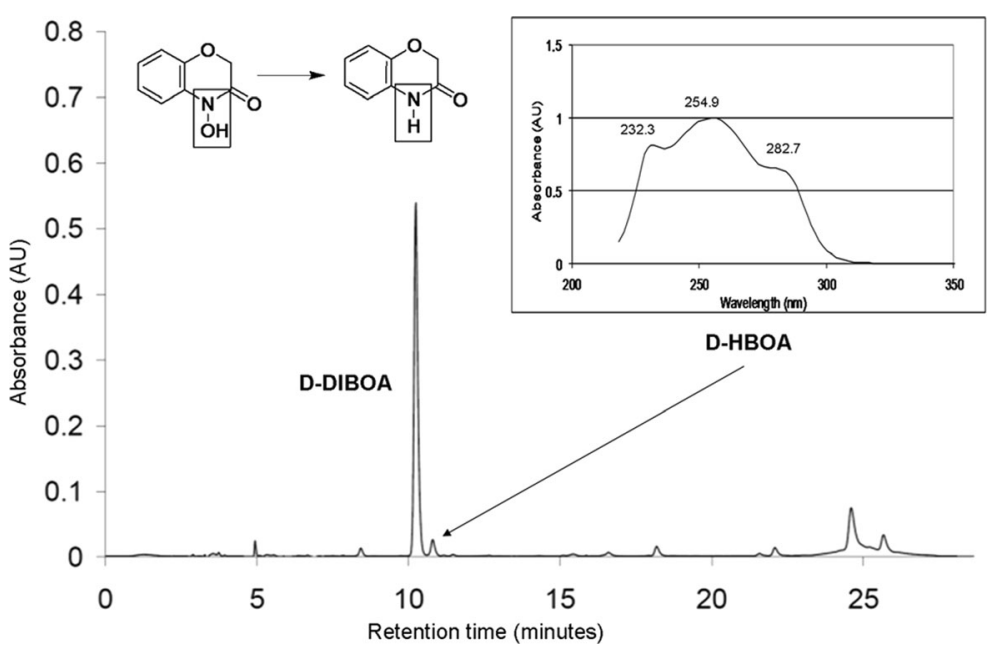




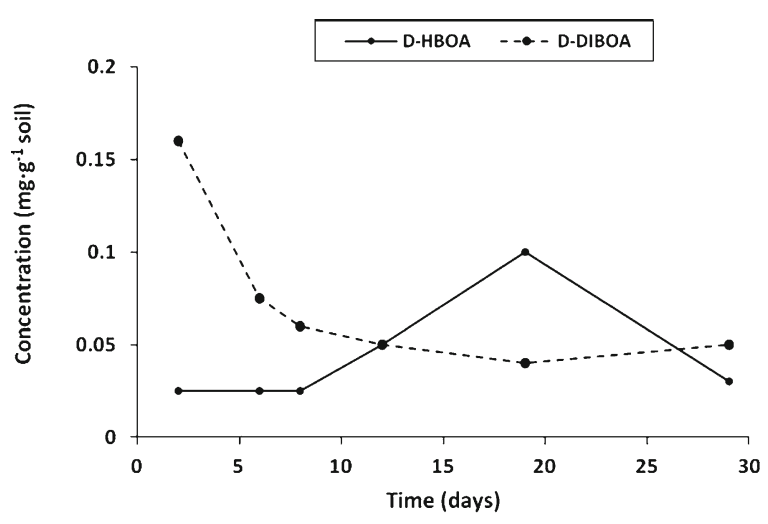

Fig. 6 Comparison of D-DIBOA and D-HBOA concentrations in soil. Values shown for sampling times of $2,6,8,12,19$, and 30 days

of D-HBOA decreased rapidly. Other unknown metabolites appeared after day 20 (none of which belonged to the compounds used as standards) and it is therefore evident that D-HBOA is metabolized into chemicals other than those belonging to the DIBOA degradation series characterized by us (Macias et al. 2005c). DHBOA is also likely to be degraded over time, as shown in Fig. 6.

The first degradation product from D-DIBOA in wheat crop soil begins to degrade only a short time after it first appears. D-HBOA is less active than D-DIBOA (Macias et al. 2005b). Thus, this degradation process leads to a loss of phytotoxicity, at least during the initial stages.

\section{Discussion}

The biodegradability of D-DIBOA was studied by a previously reported methodology (Macias et al. 2004, 2005a). These experiments provided evidence that the analytical methodology employed for DIBOA and DIMBOA degradation studies is valid to determine the stability of D-DIBOA. A half-life of $3.6 \pm 0.2$ days was obtained and this is much higher than that previously determined at the same dosage on soil for DIBOA (26 h) (Macias et al. 2005c), DIMBOA (32 min) (Macias et al. 2004) and their benzoxazolinone derivatives (OliverosBastidas et al. 2012). This result confirms the starting hypothesis that greater stability is potentially associated with a higher stability for this 2-deoxybenzoxazinone in typical field soil. Changes in the aromatic substitution pattern modify the stability of benzoxazinones and their derivatives markedly (Macias et al. 2004, 2005c) and one would expect the half-life for D-DIBOA to be longer than that found for DIBOA and the chemicals belonging to its degradation series. Both DIBOA and its glucoside, DIBOA-Glc, had much lower degradation times at the same dose. The values were in the range $0.025-0.030 \mathrm{~h}$. The half-life of D-DIBOA is much higher than that recorded for BOA (0.26-0.31 days).

In addition, the first chemical of the D-DIBOA degradation series was determined to be the lactam DHBOA, which is slightly less phytotoxic than its precursor. This chemical also degrades and the chemicals resulting from this process are different to those previously observed in DIBOA or DIMBOA degradation studies.

Despite the fact that D-DIBOA lacks a hydroxyl group at C-2, a biodegradation process still takes place and this is promising from the point of view of its potential application as biorational pest management tool as it indicates adequate environmental compatibility.

Our findings suggest that synthetic hydroxamic acids and their derivatives may prove to be useful as novel bioherbicidal molecules. Several of these compounds, including D DIBOA, offer some residual soil activity, which may enable their eventual use as preemergent herbicides after further study.

Acknowledgments This research was supported by the Ministerio de Economía y Competitividad (MINECO), Spain (Project AGL2013-42238-R) and Consejería de Innovación, Ciencia e Industria, Junta de Andalucía, Spain (Project DP12TEP-725).

Conflict of interest The authors declare no conflict of interest associated with this manuscript.

\section{References}

Argandoña VH, Corcuera LJ, Niemeyer HM, Campbell BC (1983) Toxicity and feeding deterrency of hydroxamic acids from Gramineae in synthetic diets against the greenbug, Schizaphis graminum. Entomol Exp Appl 34:134-138

Barnes JP, Putnam AR (1987) Role of benzoxazinones in allelopathy by rye (Secale cereale L.). J Chem Ecol 13:889-906

Bravo HR, Lazo W (1996) Antialgal and antifungal activity of natural hydroxamic acids and related compounds. J Agric Food Chem 44:1569-1571

Cipollini D, Rigsby CM, Barto EK (2012) Microbes as targets and mediators of allelopathy in plants. J Chem Ecol 38:714-727 
Dayan FE, Owens DK, Duke SO (2012) Rationale for a natural products approach to herbicide discovery. Pest Manag Sci 68: 519-528

Eljarrat E, Guillamon M, Seuma J, Mogensen BB, Fomsgaard IS, Olivero-Bastidas A, Macias FA, Stochmal A, Oleszek W, Shakaliene O, Barcelo D (2004) First European interlaboratory study of the analysis of benzoxazinone derivatives in plants by liquid chromatography. J Chromatogr A 1047:69-76

Fomsgaard IS, Mortensen AG, Carlsen SCK (2004) Microbial transformation products of benzoxazolinone and benzoxazinone allelochemicals: a review. Chemosphere 54: $1025-1038$

Gerwick BC, Sparks TC (2014) Natural products for pest control: an analysis of their role, value and future. Pest Manag Sci 70: 1169-1185

Macias FA, Oliveros-Bastidas A, Marin D, Castellano D, Simonet AM, Molinillo JMG (2004) Degradation studies on benzoxazinoids. Soil degradation dynamics of 2,4-dihydroxy-7-methoxy-(2 h)-1,4-benzoxazin-3(4 h)-one (DIMBOA) and its degradation products, phytotoxic allelochemicals from gramineae. J Agric Food Chem 52: 6402-6413

Macias FA, Chinchilla N, Varela RM, Oliveros-Bastidas A, Marin D, Molinillo JMG (2005a) Structure-activity relationship studies of benzoxazinones and related compounds. Phytotoxicity on Echinochloa crus-galli (L.) P. Beauv. J Agric Food Chem 53:4373-4380

Macias FA, Marin D, Oliveros-Bastidas A, Castellano D, Simonet AM, Molinillo JMG (2005b) Structure-activity relationships (SAR) studies of benzoxazinones, their degradation products and analogues. Phytotoxicity on standard target species (STS). J Agric Food Chem 53:538-548

Macias FA, Oliveros-Bastidas A, Marin D, Castellano D, Simonet AM, Molinillo JMG (2005c) Degradation studies on benzoxazinoids. Soil degradation dynamics of $(2 R)-2-\mathrm{O}-\beta-$ D-glucopyranosyl-4-hydroxy-(2H)- 1,4-benzoxazin-3(4H)one (DIBOA-Glc) and its degradation products, phytotoxic allelochemicals from Gramineae. J Agric Food Chem 53: 554-561

Macias FA, De SJM, Chinchilla N, Marin D, Varela RM, Molinillo JMG (2006a) New herbicide models from benzoxazinones: aromatic ring functionalization effects. J Agric Food Chem 54:9843-9851

Macias FA, Marin D, Oliveros-Bastidas A, Castellano D, Simonet AM, Molinillo JMG (2006b) Structure-activity relationship (SAR) studies of benzoxazinones, their degradation products, and analogues. Phytotoxicity on problematic weeds Avena fatua L. and Lolium rigidum Gaud. J Agric Food Chem 54: $1040-1048$

Macias FA, Marin D, Oliveros-Bastidas A, Chinchilla D, Simonet AM, Molinillo JMG (2006c) Isolation and synthesis of allelochemicals from gramineae: benzoxazinones and related compounds. J Agric Food Chem 54:991-1000
Macias FA, Marin D, Oliveros-Bastidas A, Molinillo JMG (2006d) Optimization of benzoxazinones as natural herbicide models by lipophilicity enhancement. J Agric Food Chem 54:9357-9365

Macías FA, Marin D, Oliveros-Bastidas A, Simonet AM, Molinillo JMG (2007) Ecological relevance of the degradation processes of allelochemicals. In: Fujii Y, Hiradate S (eds) Allelopathy: new concepts \& methodology. Science Publishers Inc, Enfield, pp 91-107

Macías FA, Chinchilla N, Varela RM, Molinillo JMG, Marín D, De Siqueira JM (2008) Modified benzoxazinones in the system Oryza sativa-Echinochloa crus-galli: an approach to the development of biorational herbicide models. J Agric Food Chem 56:9941-9948

Macias FA, Chinchilla N, Varela RM, Molinillo JMG, Marin D, de Siqueira JM (2009) Aromatic-ring-functionalised benzoxazinones in the system Oryza sativa-Echinochloa crus-galli as biorational herbicide models. Pest Manag Sci 65:1104-1113

Macias FA, Chinchilla N, Arroyo E, Molinillo JMG, Marin D, Varela RM (2010) Combined strategy for phytotoxicity enhancement of benzoxazinones. J Agric Food Chem 58:2047-2053

Macías FA, Chinchilla N, Arroyo E, Varela RM, Molinillo JMG, Marín D (2010) Multifunctionalised benzoxazinones in the systems Oryza sativa-echinochloa crus-galli and Triticum aestivum-Avena fatua as natural-product-based herbicide leads. Pest Manag Sci 66:1137-1147

Macias FA, Oliveros-Bastidas A, Marin D, Chinchilla N, Castellano D, Molinillo JMG (2014) Evidence for an allelopathic interaction between rye and wild oats. J Agric Food Chem 62:9450-9457

Meier U (1997) Growth stages of mono- and dicotyledonous plants. Blackwell Wissenschafts-Verlag, Berlin; Boston

Oliveros-Bastidas A (2006) Allelopathic studies on Gramineae. Benzoxazinoids as allelochemicals. PhD Degree, University of Cádiz

Oliveros-Bastidas AJ, Macias FA, Marin D, Carrera-Fernandez CA, Molinillo JMG (2009) Decomposition of 2,4-dihydroxy-2H-1,4-benzoxazin-3(4H)-one (DIBOA), an allelochemical of grasses. Consequences for measurements of biological activity. Quimica no Brasil 3:53-60

Oliveros-Bastidas A, Macias FA, Marin D, Molinillo JMG, Barbosa LCA, Demuner AJ (2012) Kinetics and mechanism of the degradation in soil of 2-hydroxy-(2H)-1,4-benzoxazin$3(4 \mathrm{H})$-one (HBOA), phytotoxic allelochemicals from gramineae. Av cien ing 3:47-67

Perez FJ, Ormeno-Nunez J (1991) Difference in hydroxamic acid content in roots and root exudates of wheat (Triticum aestivum L.) and rye (Secale cereale L.): possible role in allelopathy. J Chem Ecol 17:1037-1043

Schulz M, Marocco A, Tabaglio V, Macias FA, Molinillo JMG (2013) Benzoxazinoids in rye allelopathy - from discovery to application in sustainable weed control and organic farming. J Chem Ecol 39:154-174 